\title{
Propriedades físicas e mecânicas da madeira Tatajuba (Bagassa guianensis) proveniente de duas diferentes regiões brasileiras
}

\author{
Physical and mechanical properties of Tatajuba \\ wood specie (Bagassa guianensis) from two \\ different Brazilian regions
}

\author{
Tales Fernando Pegoraro Lima ${ }^{1}$, Tiago Hendrigo de Almeida ${ }^{2}$, \\ Diego Henrique de Almeida ${ }^{1}$, André Luis Christoforo ${ }^{1}$, \\ Francisco Antonio Rocco Lahr ${ }^{3}$
}

\footnotetext{
${ }^{1}$ Universidade Federal de São Carlos, Departamento de Engenharia Civil, CEP: 13565-905, São Carlos, SP, Brasil. e-mail: taepegoraro@gmail.com; diegoestruturas@gmail.com; christoforoal@yahoo.com.br

${ }^{2}$ Universidade de São Paulo, Escola de Engenharia de São Carlos, Departamento de Engenharia de Materiais, CEP: 13566-590, São Carlos, SP, Brasil.

e-mail: tiago.hendrigo@gmail.com

${ }^{3}$ Universidade de São Paulo, Escola de Engenharia de São Carlos, Departamento de Engenharia de Estruturas, CEP: 13566-590, São Carlos, SP, Brasil.

e-mail: frocco@sc.usp.br
}

\begin{abstract}
RESUMO
Como consequência dos fatores edafoclimáticos, as propriedades da madeira podem ser influenciadas de acordo com a região de desenvolvimento da árvore. O objetivo do presente trabalho consistiu em verificar a influência da região de extração de lotes da madeira de Tatajuba (Bagassa guianensis), provenientes de duas diferentes regiões do Brasil (Alta Floresta e Bonfim do Sul, municípios dos estados de Mato Grosso e Roraima, respectivamente), ao teor de umidade de $12 \%$, em algumas de suas propriedades físicas e mecânicas. Para cada um dos lotes foram determinadas duas propriedades físicas e cinco propriedades mecânicas, de acordo com a norma ABNT NBR 7190:1997. Para avaliar a influência da região de extração nas propriedades, foi realizada a análise de variância de Kruskal-Wallis, considerando resultados significativos (valores não equivalentes) aqueles que atendiam à condição $\mathrm{P}$-valor $<0,05$. De acordo com os resultados da análise estatística, os diferentes lotes da mesma espécie de madeira apresentaram valores médios das propriedades estudadas equivalentes em relação ao conjunto composto por todas as madeiras, porém, os valores médios da resistência na compressão paralela $\left(\mathrm{f}_{\mathrm{c} 0}\right)$ e da densidade aparente não foram significativos entre as duas regiões. Os valores característicos da $\mathrm{f}_{\mathrm{c} 0}$ foram diferentes para os lotes das duas regiões, ao teor de umidade de 12\%, implicando em classes de resistência diferentes (D50 - Alta Floresta e D60 - Bonfim do Sul).
\end{abstract}

Palavras-chave: Caracterização; Classes de resistência; Estruturas de madeira; Floresta Amazônica.

\section{ABSTRACT}

As a consequence of the edaphoclimatic factors, the properties of the wood can be influenced according to the site of development of the tree. The aim of the research was to verify the influence, in some physical and mechanical properties, of the extraction site of bacthes of Tatajuba wood specie (Bagassa guianensis) from two different regions of Brazil (Alta Floresta and Bonfim do Sul, Mato Grosso and Roraima Brazilian states, respectively) at $12 \%$ of moisture content. For each bacthes two physical and five mechanical properties were determined, according to ABNT NBR 7190:1997 Brazilian Code. In order to evaluate the influence of the extraction region on the properties, Kruskal-Wallis analysis of variance was performed, considering significant results (non-equivalent values) for those that met P-value $<0.05$. According to the results, the different batches of the same wood species presented equivalent average values of the studied properties in relation to the set, but compressive strength parallel to the grain and apparent density average values were not significant between the two sites. The characteristic values of compressive strength parallel to the grain were different for the different batches, at the moisture content of $12 \%$, classified the same species in different strength classes (D50 - Alta Floresta and D60 - Bonfim do Sul). 
Keywords: Amazon Forest, Characterization, Strength classes, Timber structures.

\section{INTRODUÇÃO}

A Floresta Amazônica é um dos principais biomas do mundo, abrangendo 70\% dos países da América do Sul $\left(6850476 \mathrm{~km}^{2}\right)$. Em relação à flora, a região é abundante, sendo que estudos recentes estimam que exista 16 mil espécies diferentes de árvores (no território Brasileiro, a projeção é que haja 12655) [1,2]. Para a utilização racional da madeira na construção civil (como elemento estrutural ou como parte da edificação - pisos, forros e guarnições) é requisito básico o conhecimento de suas propriedades físicas e mecânicas [3-5].

A Tatajuba (Bagassa guianensis) é uma espécie nativa brasileira proveniente da Floresta Amazônica com ocorrências no Brasil (Acre, Amazonas, Maranhão, Mato Grosso, Pará, Rondônia e Roraima), Guiana, Guiana Francesa e Suriname [6]. A Tatajuba apresenta valor médio de resistência à compressão paralela às fibras e densidade aparente da ordem de $79 \mathrm{MPa}$ e $0,9 \mathrm{~g} / \mathrm{cm}^{3}$, respectivamente [7] e ponto de saturação das fibras (PSF) da ordem de 22,8\% [8]. Esta apresenta dureza paralela e perpendicular às fibras igual a $121 \mathrm{MPa}$ e $94 \mathrm{MPa}[8]$.

A resistência à biodegradação da Tatajuba, é relacionada aos extrativos contidos no seu cerne e influenciam positivamente esta propriedade [9]. Por estas características, a Tatajuba pode ser utilizada na construção civil desde aplicações leves e internas (painéis, lambris e forros) até pesadas e externas (dormentes ferroviários, cruzetas, estacas e defensas) [6].

Das pesquisas com foco na caracterização da madeira de espécies tropicais brasileiras para fins estruturais, por ser um material de origem natural, fica evidente a variabilidade das suas propriedades, por esta razão, é importante a classificação das inúmeras espécies dentro de classes de resistência, sendo possível direcionar seu emprego na construção civil [10-15].

Esta variabilidade das propriedades da madeira pode estar relacionada com as características inerentes das diferentes espécies e gêneros de árvores que fornecem este material, além disso, por fatores anatômicos, direção das fibras, densidade e teor de umidade [16-20]. O teor de umidade da madeira é de fundamental conhecimento para a estimativa de suas propriedades físicas e mecânicas e para o seu emprego de maneira racional na construção civil. A norma ABNT NBR 7190:1997 [21] recomenda o teor de umidade de equilíbrio da madeira como $12 \%$ [22-27].

Além destes fatores, os edafoclimáticos, aqueles inerentes à região de desenvolvimento da árvore (solo, relevo, clima, temperatura, chuvas, entre outros), influenciam o desenvolvimento da árvore e, consequentemente, as propriedades da madeira [28-34].

O objetivo desta pesquisa foi verificar a influência da região de extração nas propriedades físicas e mecânicas da madeira de Tatajuba e, além disso, contribuir com as pesquisas para a utilização racional das madeiras provenientes de reservas legais da Amazônia.

\section{MATERIAIS E MÉTODOS}

Para realização desta pesquisa foram utilizados dois lotes homogêneos da madeira de Tatajuba (Bagassa guianensis) provenientes de reservas legais de duas diferentes localidades do Brasil: Alta Floresta $(A F)$, estado de Mato Grosso [0952'32"S; 56 05'10"W] e Bonfim do Sul $(B F)$, estado de Roraima [03'21'23"N; 59 49'59” W]. Os lotes de madeira de ambas localidades eram constituídos por vigas de dimensões nominais da seção transversal de $6 \mathrm{~cm}$ x $12 \mathrm{~cm}$ e comprimento de $4 \mathrm{~m}$, obtidos de produtores dos estados de Roraima e Mato Grosso. A secagem dos lotes foi realizada ao ar livre no Laboratório de Madeira e de Estruturas de Madeira (LaMEM), da Escola de Engenharia de São Carlos (EESC), Universidade de São Paulo (USP), onde as condições climáticas permitem alcançar o teor de umidade de 12\%, de acordo com a ABNT NBR 7190 (1997).

Para confecção dos corpos de prova e procedimentos de ensaios para determinação das propriedades físicas e mecânicas, foram seguidas as recomendações do Anexo B "Determinação das Propriedades das Madeiras para Projetos de Estrutura" da norma ABNT NBR 7190 (1997) [18]. Para isso, foram caracterizadas duas duas propriedades físicas e cinco propriedades mecânicas (Tabela 1), sendo fabricados 12 corpos de prova para cada uma, para ambos os lotes. 
Tabela 1: Propriedades físicas e mecânicas da madeira de Tatajuba (Bagassa guianensis).

\begin{tabular}{lc}
\hline Propriedades & Sigla \\
\hline Densidade básica & $\rho_{\mathrm{bas}}$ \\
Densidade aparente a 12\% & $\rho_{12}$ \\
Resistência à compressão paralela às fibras & $\mathrm{f}_{\mathrm{c} 0}$ \\
Resistência à tração paralela às fibras & $\mathrm{f}_{\mathrm{t} 0}$ \\
Resistência ao cisalhamento paralelo às fibras & $\mathrm{f}_{\mathrm{v} 0}$ \\
Módulo de elasticidade na compressão paralela às fibras & $\mathrm{E}_{\mathrm{c} 0}$ \\
Módulo de elasticidade na tração paralela às fibras & $\mathrm{E}_{\mathrm{t} 0}$ \\
\hline
\end{tabular}

Os valores característicos de resistência $\left(f_{k}\right)$ foram calculados colocando em ordem crescente de resistência os valores individuais de cada corpo de prova $\left(f_{1}<f_{2}<\ldots<f_{n}\right)$, desprezando-se o valor mais alto se o número de corpos de prova fosse ímpar, não se tomando para $\mathrm{f}_{\mathrm{k}}$ valor inferior a $\mathrm{f}_{1}$, nem a 0,7 do valor médio (Equação 1). A partir dos valores característicos de resistência, foi possível enquadrar os lotes nas classes de resistência recomendados pelo PN02:126.10-001-1 (NBR 7190) [36].

$f_{k}=\left(2 \frac{f_{1}+f_{2}+\ldots+f_{\frac{n}{2}-1}}{\frac{n}{2}-1}-f_{\frac{n}{2}}\right) 1,1$

Para avaliar a influência da região de extração e do teor de umidade nas propriedades físicas e mecânicas da madeira de Tatajuba, foi realizada a análise de variância (ANOVA) de Kruskal-Wallis (utilizando o software BioEstat 5.0), considerando resultados significativos (valores não equivalentes) aqueles que atendem à condição $\mathrm{P}$-valor $<0,05$.

\section{RESULTADOS E DISCUSSÕES}

As Tabelas 2 e 3 apresentam os resultados correspondentes aos lotes provenientes das regiões de Alta Floresta, Mato Grosso $(A F)$ e Bomfim do Sul, Roraima $(B F)$ nos teores de umidade de $12 \%$. As tabelas apresentam os valores médios (x), mínimos (Min) e máximos (Max) de cada uma das propriedades estudadas e os respectivos coeficientes de variação $(\mathrm{CV})$ e, também, os valores de resistência características $\left(\mathrm{f}_{\mathrm{ck}}\right)$.

Tabela 2: Propriedades do lote Tatajuba proveniente de Alta Floresta (teor de umidade de 12\%).

\begin{tabular}{lccccc}
\hline Propriedades & $\mathrm{x}$ & $\mathrm{CV}(\%)$ & $\operatorname{Min}$ & $\operatorname{Max}$ & $\mathrm{f}_{\mathrm{k}}(\mathrm{MPa})$ \\
\hline$\rho_{\mathrm{bas}}\left(\mathrm{g} / \mathrm{cm}^{3}\right)$ & 0,75 & 13,92 & 0,53 & 1,03 & - \\
$\rho_{12}\left(\mathrm{~g} / \mathrm{cm}^{3}\right)$ & 0,81 & 13,83 & 0,61 & 1,07 & - \\
$\mathrm{f}_{\mathrm{c} 0}(\mathrm{MPa})$ & 69 & 15,46 & 41 & 78 & 55 \\
$\mathrm{f}_{\mathrm{t} 0}(\mathrm{MPa})$ & 91 & 37,95 & 39 & 148 & 64 \\
$\mathrm{f}_{\mathrm{v} 0}(\mathrm{MPa})$ & 18 & 23,28 & 11 & 25 & 12 \\
$\mathrm{E}_{\mathrm{c} 0}(\mathrm{MPa})$ & 18528 & 18,07 & 12893 & 24202 & - \\
$\mathrm{E}_{\mathrm{t} 0}(\mathrm{MPa})$ & 18284 & 10,89 & 13999 & 20884 & - \\
\hline
\end{tabular}


Tabela 3: Propriedades do lote Tatajuba proveniente de Bonfim do Sul (teor de umidade de 12\%).

\begin{tabular}{lccccc}
\hline \multicolumn{1}{c}{ Propriedades } & $\mathrm{x}$ & $\mathrm{CV}(\%)$ & Min & Max & $\mathrm{f}_{\mathrm{k}}(\mathrm{MPa})$ \\
\hline$\rho_{\mathrm{bas}}\left(\mathrm{g} / \mathrm{cm}^{3}\right)$ & 0,74 & 5,48 & 0,67 & 0,81 & - \\
$\rho_{12}\left(\mathrm{~g} / \mathrm{cm}^{3}\right)$ & 0,94 & 6,07 & 0,83 & 1,06 & - \\
$\mathrm{f}_{\mathrm{c} 0}(\mathrm{MPa})$ & 80 & 18,62 & 45 & 99 & 60 \\
$\mathrm{f}_{\mathrm{t} 0}(\mathrm{MPa})$ & 93 & 22,5 & 48 & 127 & 65 \\
$\mathrm{f}_{\mathrm{v} 0}(\mathrm{MPa})$ & 20 & 8,53 & 17 & 23 & 18 \\
$\mathrm{E}_{\mathrm{c} 0}(\mathrm{MPa})$ & 18571 & 17,47 & 10566 & 24425 & - \\
$\mathrm{E}_{\mathrm{t} 0}(\mathrm{MPa})$ & 17319 & 12,05 & 14292 & 21940 & - \\
\hline
\end{tabular}

A densidade aparente do lote proveniente de Alta Floresta é significativamente menor quando comparada com os trabalhos de Dias e Lahr (2004) [7], Almeida et al. (2017) [8] e Lahr et al. (2010) [37], apresentando um valor médio de $0,81 \mathrm{~g} / \mathrm{cm}^{3}$, e podendo ser considerada uma madeira pesada. O lote proveniente de Bonfim do Sul apresentou densidade aparente igual a $0,94 \mathrm{~g} / \mathrm{cm}^{3}$.

A norma ABNT NBR 7190 (1997) [21] não faz menção a respeito da região de extração da madeira, porém, como é mostrado nesta pesquisa, para uma mesma espécie, valores médios de resistência obtidos para diferentes lotes, podem apresentar valores diferentes de resistência característica, o que influencia diretamente o dimensionamento de estruturas de madeira.

A Tabela 4 apresenta os P-valores da ANOVA de Kruskal-Wallis (valores considerados significativos (P-valor $<5 \%$ ) estão sublinhados) para cada propriedade analisada confrontando os resultados da região de Alta Floresta com os resultados de Bonfim do $\mathrm{Sul}(A F \mathrm{x} B S)$ e os resultados de cada região com o conjunto de todos os resultados $(A F \times\{A F ; B S\}$ e $B S \times\{A F ; B S\})$. Os valores de $\rho_{12}$ e $\mathrm{f}_{\mathrm{c} 0}$ dos lotes de Alta Floresta e Bonfim do Sul não apresentam valores médios equivalentes. Entretanto, todas as propriedades dos diferentes lotes estudados nesta pesquisa, para a madeira de Tatajuba são equivalentes ao conjunto dos resultados.

Tabela 4: P-valores da ANOVA de Kruskal-Wallis confrontando as diferentes regiões de origem.

\begin{tabular}{cccc}
\hline Propriedades & $A F \times B S$ & $A F \times\{A F ; B S\}$ & $B S \times\{A F ; B S\}$ \\
\hline$\rho_{\text {bas }}$ & 0,7948 & 0,8798 & 0,8796 \\
$\rho_{12}$ & $\underline{0,0055}$ & 0,1068 & 0,1057 \\
$\mathrm{f}_{\mathrm{c} 0}$ & $\underline{0,0066}$ & 0,1144 & 0,1143 \\
$\mathrm{f}_{\mathrm{t} 0}$ & 0,7728 & 0,8666 & 0,8666 \\
$\mathrm{f}_{\mathrm{v} 0}$ & 0,0729 & 0,2976 & 0,2956 \\
$\mathrm{E}_{\mathrm{c} 0}$ & 0,9081 & 0,9464 & 0,9464 \\
$\mathrm{E}_{\mathrm{t} 0}$ & 0,2253 & 0,4806 & 0,4806 \\
\hline
\end{tabular}

\section{CONCLUSÃO}

Foi possível concluir, através dos resultados obtidos da presente pesquisa, que:

- A densidade aparente e a resistência à compressão paralela às fibras dos lotes de Alta Floresta e Bonfim do Sul não são equivalentes entre si, porém são equivalentes quando comparadas ao conjunto de todos os dados disponíveis;

- O lote de Alta Floresta foi enquadrado na classe de resistência D50, enquanto que o lote de Bonfim do Sul foi enquadrado na classe D60. Ainda assim, cada um deles possui resultados estatisticamente equivalentes ao conjunto de todos os resultados disponíveis;

- É necessário realizar mais pesquisas (com outras espécies tropicais brasileiras e com outras propriedades físicas e mecânicas) que considerem a consideração a região de extração da madeira para fins estruturais.

\section{AGRADECIMENTOS}

Por todo o apoio fornecido, os autores agradecem a Coordenação de Aperfeiçoamento de Nível Superior (CAPES) e ao Conselho Nacional de Desenvolvimento Científico e Tecnológico (CNPq). 


\section{BIBLIOGRAFIA}

[1] BAENA, M. S, S., GARCIA, L. C., PETERSON, A. T. "Completeness of digital accessible knowledge of the plants of Brazil and priorities for survey and inventory", Diversity and Distributions, v. 20, pp. 369-381, 2014.

[2] STEEGE, H., VAESSEN, R. W., LÓPEZ, D. C., et al., "The discovery of the Amazonian tree flora with an update checklist of all known tree taxa", Scientific Reports, v. 6, n. 29549, pp. 1-15, 2016.

[3] CALIL JUNIOR, C., LAHR, F. A. R., DIAS, A. A., Dimensionamento de elementos estruturais de madeira, Barueri, Manole, 2003.

[4] NAHUZ, A. R., Catálogo de madeiras brasileiras para a construção civil, São Paulo, IPT, 2013.

[5] ALMEIDA, D. H., DIAS, A. A., "Comparison between test methods to determine wood embedment strength parallel to the grain”, Revista Árvore, v. 40, n. 4, pp. 741-748, 2016.

[6] INSTITUTO DE PESQUISAS TECNOLÓGICAS, IPT,

http://www.ipt.br/informacoes_madeiras3.php?madeira=50. Acessado em julho de 2017.

[7] DIAS, F. M., LAHR, F. A. R., "Estimativa de propriedades de resistência e rigidez da madeira através da densidade aparente", Scientia Forestalis, v. 65, pp. 102-113, 2004.

[8] ALMEIDA, T. H., CHRISTOFORO, A. L., LAHR, F. A. R., Study of dimensional stability of Brazilian tropical wood species, Chisinau/Balti, Lambert Academic Publishing, 2017.

[9] ROYER, M., RODRIGUES, A. M. S., HERBETTE, G., et al., "Efficacy of Bagassa guianensis Aubl. extract against wood decay and human pathogenic fungi", International Biodeterioration and Biodegradation, v. 70, pp. 55-59, 2012.

[10] ALMEIDA, D. H., SCALIANTE, R. M., MACEDO, L. B., et al., "Caracterização completa da madeira da espécie amazônica Paricá (Schizolobium amazonicum HERB) em peças de dimensões estruturais", Revista Árvore, v. 37, n. 6, pp. 1175-1181, 2013.

[11] LAHR, F. A. R., AFTIMUS, B. H. C., ARROYO, F. N., et al., "Full characterization of Vatairea $s p$ wood specie”, International Journal of Materials Engineering, v. 6, n. 3, pp. 92-96, 2016.

[12] LAHR, F. A. R., ARROYO, F. N., ALMEIDA, T. H., et al., "Full characterization of Erisma encinatum Warm wood specie", International Journal of Materials Engineering, v. 6, n. 5, pp. 147-150, 2016.

[13] CHRISTOFORO, A. L., AFTIMUS, B. H. C., PANZERA, T. H., et al., "Physical-mechanical characterization of the Anandenanthera colubrine wood specie”, Engenharia Agrícola, v. 37, n. 2, pp. 376-384, 2017.

[14] CHRISTOFORO, A. L., ARROYO, F. N., SILVA, D. A. L., et al., "Full characterization of Calycophyllum multiflorum wood specie”, Engenharia Agrícola, v. 37, n. 4, pp. 637-643, 2017.

[15] AlMEIDA, T. H.; ALMEIDA, D. H.; ARAÚJO, V. A.; SILVA, S. A. M.; CHRISTOFORO, A. L.; LAHR, F. A. R., "Density as estimator of dimensional stability quantities of Brazilian tropical woods", BioResouces, v. 12, n. 3, pp. 6579-6590, 2017.

[16] ALMEIDA, D. H., SCALIANTE, R. M., CHRISTOFORO, A. L., et al., "Tenacidade da madeira como função da densidade aparente", Revista Árvore, v. 38, n. 1, pp. 203-207, 2014.

[17] ALMEIDA, D. H., CHAHUD, E., ALMEIDA, T. H., et al., "Determination of density, shear and compression parallel to the grain strengths of Pariri (Pouteria sp.), Brazilian native wood specie", International Journal of Materials Engineering, v. 5, n. 1, pp. 109-112, 2015.

[18] CAVAlHEIRO, R. S., ALMEIDA, D. H., ALMEIDA, T. H., et al., "Mechanical properties of Paricá wood using structural members and clear specimens", International Journal of Materials Engineering, v. 6 , n. 2, pp.56-59., 2016.

[19] CAVAlHEIRO, R. S., ALMEIDA, D. H., ALMEIDA, T. H., et al., "Density as estimator of shrinkage for some Brazilian wood species", International Journal of Materials Engineering, v. 6, n. 3, pp.107-112, 2016.

[20] ALMEIDA, T. H., ALMEIDA, D. H., CHRISTOFORO, A. L., et al., "Density as estimator of strength in compression parallel to the grain strength", International Journal of Materials Engineering, v. 6, n. 3, pp. 67-71, 2016.

[21] ASSOCIAÇÃO BRASILEIRA DE NORMAS TÉCNICAS. ABNT NBR 7190: Projeto de estruturas de madeira. Rio de Janeiro: ABNT, 1997.

[22] STOLF, D. O., BERTOLINI, M. S., FERRO, F. S., et al., "Influência do teor de umidade ma proprieda- 
de de tenacidade de espécies florestais", Floresta e Ambiente, v. 21, n. 4, pp. 501-508, 2014.

[23] YAMASAKI, M., TSUZUKI, C., SASAKI, Y., et al., "Influence of moisture content on estimating Young's modulus of full-scale timber using stress wave velocity", Journal of Wood Science, v. 63, n. 3, pp. 225-235.

[24] MOTTA, J. P., OLIVEIRA, J. T. S., ALVES, R. C., "Influência do teor de umidade nas propriedades de adesão da madeira de Eucalipto", Construindo, v. 4, n. 2, pp. 96-103, 2012.

[25] ALMEIDA, D. H., FERRO, F. S., ICIMOTO, F. H., et al., "Determinação da rigidez de Pinus elliottii em diferentes teores de umidade por meio de ensaios mecânicos não destrutivos", Scientia Forestalis, v. 44, n. 110, pp. 303-309, 2016.

[26] MAJANO, A. M.; HUGHES, M.; CABO, J. F. L., "The fracture toughness and properties of thermally modified beech and ash at different moisture contents", Wood Science and Technology, v. 46, n. 1-3, pp. 521, 2012.

[27] MATOS, G. S., MOLINA, J. C., "Resistência da madeira ao cisalhamento paralelo às fibras segundo as normas ABNT NBR 7190:1997 e ISO 13910:2005”, Revista Matéria, v. 22, n. 4, pp. 1069-1079, 2016.

[28] CARVALHO, M. S. P., CARVALHO, J. O. P. "Influência do espaçamento na densidade da madeira de Bagassa guianensis Aubl. (Tatajuba) no planalto de Belterra, Pará", Revista Ciências Agrárias, v. 35, pp. 7378, 2001.

[29] MORAES NETO, S. P., TELES, R. F., RODRIGUES, T. O., et al., Propriedades mecânicas da madeira de cinco procedências de Pinus caribaea var. hondurensis implantadas no cerrado do Distrito Federal, DF, Planaltina, Embrapa, 2009.

[30] LIMA, I. L., LONGUI, E. L., ANDRADE, I. M., et al., "Efeito da procedência em algumas propriedades da madeira de Gallesia integrifolia (Spreng.) Harms", Revista do Instituto Florestal, v. 22, n. 1, pp. 6169, 2010.

[31] ROMAGNOLI, M.; CAVALLI, D.; SPINA, S., "Wood quality of Chestinut: relationship between ring width, specific gravity, and physical and mechanical properties", BioResources, v. 9, n. 1, pp. 1132-1147, 2014.

[32] HUDA, A. S. M. A., KOUBAA, A., CLOUTIER, A., et al., "Variation of the physical and mechanical properties of hybrid poplar clones", BioResources, v. 9, n. 1, pp. 1456-1471, 2014.

[33] CSORDÓS, D., NÉMETH, R., BAK. M., "Variation of colour properties between and within new Robinia varities with enhanced growing rates from different sites", BioResources, v. 9, n. 4, pp. 7099-7108, 2014.

[34] CUECUECHA, O. G. V., CAMPOS, E. M. Z., GALLEGOS, E. G., et al., "Densidad básica de la madera de los pinos y su relación con propriedades edáficas", Maderas y Bosques, v. 21, n. 1, pp. 129-138, 2015.

[35] CORAL, L. L. T., GUEVARA, J. E. O., WEBER, J. C., et al., "Variation in wood physical properties within stems of Guazuma crinita, a timber tree species in the Peruvian Amazon", Maderas y Bosques, v. 23, n. 1, pp. 53-61, 2017.

[36] ASSOCIAÇÃO BRASILEIRA DE NORMAS TÉCNICAS. ABNT NBR 7190: PN02:126.10-001-1: Projeto de estruturas de madeira. Rio de Janeiro: ABNT, 2011.

[37] LAHR, F. A. R., CHAHUD, E., FERNANDES, R. A., et al., "Influência da densidade na dureza paralela e na dureza normal às fibras para algumas espécies tropicais brasileiras", Scientia Forestalis, v. 38, n. 86, pp. 153-158, 2010. 